\title{
El agotamiento emocional está asociado con factores estresantes relacionados con el trabajo: Estudio multicéntrico y transversal en hospitales públicos de Malasia \\ Emotional exhaustion is associated with work related stressors: a cross-sectional multicenter study in Malaysian public hospitals
}

\author{
Dr. Erwin J. Khoo a, Dr. Sami Aldubai ${ }^{b}$, Dr. Kurubaran Ganasegeran ${ }^{c}$, \\ Estud. Bernice X. E. Leed, Estud. Nurul A. Zakariad y Dr. Kah K. Tan
}

a. Departamento de Pediatría, Universidad Médica Internacional, Malasia.

b. Facultad deMedicina, SEGI University, Selangor, Malasia.

c. Centro de

Investigación Clínica, Hospital Seberang Jaya, Penang, Malasia.

d. Facultad deMedicina, Universidad Médica Internacional, Malasia.

e. Departamento de Pediatría, Hospital Tuanku Ja'afar, Seremban, Malasia.

Correspondencia: Dr. Erwin J. Khoo: jiayuan_khoo@imu. edu.my

\section{Financiamiento:} Los fondos para este estudio y para los incentivos en efectivo que se entregaron a los sujetos como muestra de agradecimiento provienen de una beca para investigación de la Universidad Médica Internacional

(CSc/Sem6(36)2014).

Conflicto de intereses: En el momento de la presentación, Tan Kah Kee era el director del Departamento de Pediatría en el hospital Tuanku Ja'afar Seremban.

Recibido: 13-6-2016 Aceptado: 24-10-2016

\section{RESUMEN}

Introducción. El agotamiento emocional es un componente importante del síndrome de burnout (desgaste profesional). Elburnout es común entre los médicos. Afecta su salud física y mental, su rendimientoyla calidad dela atención quebrindan. Este estudio tuvo como propósito investigar el nivel y los factores asociados con el agotamiento emocional en los médicos pediatras, en Malasia. Poblaciónymétodos. Enesteestudiomulticéntrico y transversal se utilizó un cuestionario autoadministrado, que incluía preguntas acerca de las características sociodemográficas y laborales, el agotamiento emocional, la escala de estrés percibido y las fuentes laborales de estrés. Con el software SPSS, se llevaron a cabo análisis descriptivos, univariantes y multivariantes. Resultados. Un total de 197 médicos de los departamentos de pediatría de ocho hospitales respondieron el cuestionario. El $25,4 \%$ y el $24,4 \%$ de los médicos informaron, respectivamente, agotamiento emocional alto y moderado. En el análisis bivariante, 29 de las 38 opciones correspondientes a fuentes de estrés mostraron una asociación importante con el agotamiento emocional $(p<0,05)$. En el análisis multivariante, los predictores importantes de agotamiento emocional fueron: puntajes más altos en la escala del estrés percibido, abordaje de problemas psicosociales de los pacientes, falta de cortesía en las interacciones con colegas/subordinados, falta de reconocimiento de parte delos superiores, falta de incentivos y promociones, trabajo bajo presión del tiempo y necesidad de cumplir con los plazos, y establecimiento de metas inaccesibles o autoimposición de ese tipo de metas $(p<0,05)$. La fuente de estrés mencionada con mayor frecuencia fue el trato con padres difíciles $(80,2 \%)$. Conclusiones. El agotamiento emocional está asociado con fuentes de estrés en el entorno laboral pero no con factores sociodemográficos. Palabras clave: sindrome de burnout, profesional, pediatría, estrés, entorno laboral.

http:/ / dx.doi.org/10.5546/ aap.2017.212

Texto completo en inglés:

http:/ / dx.doi.org/10.5546/ aap.2017.eng.212

Cómo citar: Khoo EJ, Aldubai S, Ganasegeran K, et al. El agotamiento emocional está asociado con factores estresantes relacionados con el trabajo: Estudio multicéntrico y transversal en hospitales públicos de Malasia. Arch Argent Pediatr 2017;115(3):212-219.

\section{INTRODUCCIÓN}

El burnout, una afección relacionada con el agotamiento emocional que afecta a los profesionales que están en contacto con la gente, ${ }^{1}$ es un riesgo profesional importante en los servicios clínicos de los hospitales.

Se sabe que el burnout es un problema serio en los departamentos de pediatría. ${ }^{2-6}$ Hay muchos factores supuestos, como el estrés que generan los cuidados intensivos neonatales, ${ }^{7}$ los adolescentes desafiantes, los conflictos por la custodia de los menores, el abuso de menores ${ }^{8}$ y la frecuente necesidad de interactuar y comunicarse cuidadosamente con los pacientes y sus preocupadas familias. Los subespecialistas que trabajan en pediatría también presentan un nivel de estrés que genera gran inquietud. ${ }^{9-11}$ Si bien muchos estudios publicados han descrito la prevalencia del burnout en los contextos de pediatría, hasta donde sabemos, los factores que contribuyen al burnout no se han documentado.

Este tipo de desasosiego moral puede llevar a una atención profesional de calidad deficiente, una mala relación entre el médico y el paciente, menor empatía, ${ }^{12}$ más errores médicos o errores más frecuentes, ${ }^{13}$ insatisfacción con el trabajo, desgaste profesional, conflictos entre colegas, ${ }^{14}$ consumo de drogas ${ }^{15}$ e, incluso, suicidio (o intento de suicidio). ${ }^{16-17}$ Estos factores pueden comprometer la eficacia del sistema de atención de la salud y exponer a los médicos a demandas judiciales. 
Dado que los niños continúan siendo uno de los grupos de pacientes más vulnerables en los hospitales, ${ }^{18}$ es imprescindible que se tomen medidas que permitan administrar una atención pediátrica óptima para garantizar que estos profesionales de la salud estén en condiciones funcionales de ofrecer la mejor calidad de atención a los niños.

El objetivo de este estudio multicéntrico fue determinar la prevalencia del agotamiento emocional en los pediatras (desde los médicos residentes hasta los especialistas) y averiguar cuáles son los factores asociados. Nuestra hipótesis fue que el agotamiento emocional está vinculado con distintos factores del entorno laboral. Si estos factores pueden evitarse o atenuarse, podría mejorarse la calidad de atención médica que se brinda a los niños y podrían superarse algunas de las percepciones negativas de la pediatría como carrera.

\section{POBLACIÓN Y MÉTODOS Contexto y población del estudio}

La atención pediátrica en Malasia es principalmente responsabilidad del Ministerio de Salud. Se implementa un sistema universal de atención de la salud que coexiste con el sistema privado, utilizado, más que nada, para procedimientos pediátricos optativos. En 2014, la población de Malasia era de 29,9 millones de personas aproximadamente; el $25 \%$ eran menores de 14 años, ${ }^{19}$ y la cantidad de pediatras matriculados en el país desde 2001 era de 748 , lo que equivale a un pediatra cada 10000 niños. $^{20}$

La mayor parte de la atención aguda de la salud infantil secundaria en el país se cubre en 53 hospitales públicos que cuentan con pediatras. Estos hospitales aceptan niños menores de 12 años y, en el caso de algunas enfermedades infantiles crónicas, hasta los 18 años. La tasa de mortalidad infantil es de $6 / 1000$ recién nacidos vivos, comparable con la de países europeos. ${ }^{19}$

Este estudio multicéntrico transversal se llevó a cabo en ocho hospitales públicos de Malasia. Se utilizó una estrategia deliberada de casos característicos y variación máxima para asegurar la selección de una muestra representativa a nivel nacional de departamentos de pediatría en hospitales públicos. Este tipo de estrategia de muestreo se centró en (1) la distribución geográfica y la densidad de población del país (un hospital de la región del norte de Malasia Occidental; un hospital de Malasia Oriental; dos hospitales de la región del sur de Malasia
Occidental; y cuatro hospitales de la región central de Malasia Occidental en donde las poblaciones son las más densas del país); (2) sus hospitales estatales de atención especializada por derivación designados; (3) el número de camas de hospital, y (4) hospitales con capacitación de graduados que están haciendo la residencia. Incluimos en el estudio multicéntrico transversal a todos los médicos dependientes del Ministerio de Salud que había en esos departamentos de pediatría, es decir, todos los residentes, médicos y especialistas en pediatría. Se excluyeron aquellos sujetos con menos de dos meses de experiencia activa en pediatría clínica y aquellos que no tuvieran contacto con pacientes pediátricos. También se excluyó a aquellos médicos vinculados al hospital que fueran empleados por una universidad o un centro académico (no dependientes del Ministerio de Salud).

\section{Gestión de la encuesta}

Mediante cartas oficiales, se invitó a participar a los directores de los departamentos de los ocho hospitales que se incorporaron a la muestra. Se obtuvo el respaldo de organizaciones nacionales en la especialidad, y se incluyeron cartas de presentación en correos específicos. Se identificó a un pediatra de cada hospital, al que, en una charla telefónica, se le explicaron el objetivo y el procedimiento del estudio. Los cuestionarios con el consentimiento, instrucciones sencillas y una hoja informativa se enviaron por correo privado al pediatra seleccionado junto con un sobre con el franqueo pago para enviar la respuesta, excepto en el caso de los cuatro hospitales de la región central de Malasia Occidental. Luego, el pediatra seleccionado distribuyó el formulario de consentimiento y los cuestionarios a todos los médicos del departamento durante las siguientes sesiones, en las que se reunieron todos los médicos: sesiones anatomoclínicas, encuentros para el traspaso del turno y reuniones departamentales. Se entregaron a todos los participantes, de manera escrita y oral, las instrucciones habituales para completar la encuesta e indicaciones sobre la recopilación anónima de los datos. Todos los participantes firmaron un formulario de consentimiento en el momento en que se distribuyó el cuestionario. Se les pidió que ingresaran su número de registro en el Consejo Médico de Malasia. Este número se ingresó en la base de datos del registro para verificar la validez de los respondedores. Inmediatamente al finalizar cada sesión, se recopilaban las encuestas ya completas, 
y al cabo de cuatro semanas fueron enviadas de regreso a los autores por correo privado. En los departamentos de pediatría de la región central de Malasia Occidental se usó un método directo, y dos coautores distribuyeron y recogieron las encuestas personalmente. Responder a la encuesta era opcional. No obstante, a fin de alentar la participación, se entregó a los médicos un incentivo en efectivo de MYR 5 (USD 1,20) al terminar la encuesta, como muestra de agradecimiento.

\section{Instrumentos}

Se utilizó un cuestionario autoadministrado que estaba compuesto de cuatro partes. La primera parte incluía preguntas sobre las características sociodemográficas y laborales (sexo, edad, origen étnico, estado civil, cargo, título universitario, cantidad promedio de llamadas por mes y tiempo de experiencia laboral). El resto de las partes incluían la subescala de agotamiento emocional (AE), las fuentes de estrés laboral percibidas y la escala de 10 puntos de estrés percibido (EEP-10), previamente validadas en un entorno local. ${ }^{21-24}$
Medimos el agotamiento emocional utilizando la subescala de AE del cuestionario validado Maslach Burnout Inventory, en su versión para servicios humanos (MBI-HSS). La subescala AE consiste de 9 enunciados que los entrevistados calificaron con una escala Likert de 7 puntos (de 0 [nunca] a 6 [a diario]) según la frecuencia con que experimentaban esos sentimientos. Un puntaje más alto indicó un AE mayor y, por ende, un nivel más alto de burnout emocional. ${ }^{21}$ Este instrumento se validó en muchos estudios anteriores. El coeficiente alfa de Cronbach de la subescala de AE informado aquí fue de 0,93. La estructura factorial de la bibliografía arrojó un factor para los nueve enunciados de la subescala de AE. En este estudio, el análisis factorial exploratorio del AE arrojó un factor con un valor dado mayor que $1(6,3)$. La solución unifactorial fue responsable del $69,692 \%$ de la varianza.

Las fuentes percibidas de estrés laboral se evaluaron usando 38 opciones reunidas a través de una revisión bibliográfica. Se preguntó a los encuestados: ¿Cree que el siguiente aspecto es para usted una fuente de estrés? Para cada opción,

TABla 1: Características sociodemográficas de los respondedores y su asociación con el agotamiento emocional ( $n=197)$

\begin{tabular}{|c|c|c|c|}
\hline Características & $\mathbf{N}(\%)$ & Media (DE) & Valor $p$ \\
\hline \multicolumn{4}{|l|}{ Sexo } \\
\hline Masculino & $54(27,4)$ & $19,9(12,2)$ & \multirow[t]{2}{*}{0,328} \\
\hline Femenino & $143(72,6)$ & $18,0(11,4)$ & \\
\hline \multicolumn{4}{|l|}{ Edad } \\
\hline Menores de 30 años & $121(61,4)$ & $17,7(11,1)$ & \multirow[t]{2}{*}{0,210} \\
\hline 30 años o más & $76(38,6)$ & $19,9(12,4)$ & \\
\hline \multicolumn{4}{|l|}{ Origen étnico } \\
\hline Malasio & $93(47,2)$ & $19,4(11,3)$ & \multirow[t]{3}{*}{0,126} \\
\hline Chino & $76(38,6)$ & $19,0(11,0)$ & \\
\hline Indio & $28(14,2)$ & $14,4(13,7)$ & \\
\hline \multicolumn{4}{|l|}{ Estado civil } \\
\hline Soltero & $94(47,7)$ & $18,0(10,8)$ & \multirow[t]{2}{*}{0,570} \\
\hline Casado/en concubinato & $103(52,3)$ & $19,0(12,4)$ & \\
\hline \multicolumn{4}{|l|}{ Cargo } \\
\hline Residente & $74(37,6)$ & $18,5(11,1)$ & \multirow[t]{3}{*}{0,741} \\
\hline Médico & $93(47,2)$ & $18,1(11,9)$ & \\
\hline Especialista en pediatría & $30(15,2)$ & $20,0(12,5)$ & \\
\hline \multicolumn{4}{|l|}{ Graduado en la universidad } \\
\hline En el extranjero & $80(40,6)$ & $16,9(10,1)$ & \multirow[t]{2}{*}{0,092} \\
\hline En el país & $117(59,4)$ & $19,6(12,5)$ & \\
\hline \multicolumn{4}{|c|}{ Promedio de guardias por mes } \\
\hline 4 o menos & $68(34,5)$ & $17,5(10,7)$ & \multirow[t]{2}{*}{0,374} \\
\hline 5 o más & $129(65,5)$ & $19,0(12,1)$ & \\
\hline \multicolumn{4}{|c|}{ Tiempo de experiencia en pediatría } \\
\hline Menos de 2 años & $122(61,9)$ & $17,3(10,9)$ & \multirow[t]{2}{*}{0,074} \\
\hline 2 años o más & $75(38,1)$ & $20,5(12,6)$ & \\
\hline
\end{tabular}


había dos respuestas posibles: Sí o No. Algunos ejemplos de las fuentes de estrés incluidas en este estudio son temor a cometer errores médicos, exceso de trabajo durante las guardias y horarios prolongados de trabajo, compromisos yobligaciones familiares y entorno laboral hostil. Estas preguntas no se analizaron colectivamente sino de manera individual. El coeficiente alfa de Cronbach correspondiente fue 0,874 .
El estrés percibido se midió con la escala EEP-10, que permite evaluar el grado de estrés percibido por cada persona. ${ }^{22}$ Se pidió a los participantes que respondieran a cada pregunta en una escala Likert de 5 puntos (de 0 [nunca] a 4 [muy frecuentemente]) para indicar con qué frecuencia se habían sentido estresados durante el mes anterior. Los puntajes se extendían de 0 a 40, donde los más altos indicaban un mayor

TABLA 2: Asociación entre el agotamiento emocional y las fuentes percibidas de estrés laboral entre los respondedores ( $n=197)$

\begin{tabular}{|c|c|c|c|}
\hline \multirow[t]{2}{*}{ Fuentes de estrés laboral } & \multicolumn{2}{|c|}{$\begin{array}{c}\text { Media del agotamiento } \\
\text { emocional (DE) }\end{array}$} & \multirow[t]{2}{*}{$\begin{array}{c}\text { valor } \\
p\end{array}$} \\
\hline & Sí & No & \\
\hline Atención de niños con cuadros agudos en cuidados intensivos & $19,7(11,0)$ & $16,0(12,7)$ & 0,051 \\
\hline Atención de niños con cuadros crónicos & $21,1(11,9)$ & $16,2(10,9)$ & 0,003 \\
\hline Salas repletas de pacientes & $20,7(11,4)$ & $13,4(10,7)$ & $<0,001$ \\
\hline Trato con padres difíciles & $19,3(11,5)$ & $15,5(11,7)$ & 0,072 \\
\hline Abordaje de temas éticos, como desafíos de la etapa final de la vida & $20,5(11,8)$ & $15,5(10,8)$ & 0,003 \\
\hline Abordaje de problemas psicosociales de los pacientes & $21,0(11,4)$ & $15,8(11,3)$ & 0,002 \\
\hline Procedimientos complicados en niños & $21,0(11,0)$ & $16,7(12,2)$ & 0,048 \\
\hline Falta de cortesía en las interacciones con colegas / subordinados & $20,6(11,4)$ & $15,6(11,4)$ & 0,003 \\
\hline Relaciones afectivas & $21,0(11,8)$ & $17,4(11,4)$ & 0,051 \\
\hline Compromisos y obligaciones familiares & $20,6(11,8)$ & $16,8(11,2)$ & 0,020 \\
\hline Temor a contraer infecciones & $22,2(12,2)$ & $17,2(11,2)$ & 0,010 \\
\hline Temor a cometer errores médicos & $21,5(11,7)$ & $13,8(9,8)$ & $<0,001$ \\
\hline Temor a tener problemas legales relacionados con el ejercicio de la medicina & $20,6(11,8)$ & $13,6(9,7)$ & $<0,001$ \\
\hline $\begin{array}{l}\text { Sensación de que el nivel de conocimiento no es suficiente } \\
\text { para satisfacer las demandas laborales }\end{array}$ & $19,7(11,2)$ & $15,5(12,3)$ & 0,034 \\
\hline Sensación de que el salario es insuficiente & $21,8(11,4)$ & $16,0(11,2)$ & $<0,001$ \\
\hline Dudas respecto de la orientación de su carrera profesional & $22,3(11,3)$ & $15,3(11,0)$ & $<0,001$ \\
\hline Sensación de inseguridad durante el trabajo & $23,6(12,4)$ & $17,4(11,2)$ & 0,008 \\
\hline Dificultades económicas & $21,9(13,0)$ & $17,6(11,1)$ & 0,059 \\
\hline Pena o culpa por la muerte de un paciente o por resultados no satisfactorios & $18,7(12,0)$ & $18,4(11,4)$ & 0,862 \\
\hline Entorno laboral hostil & $22,8(10,6)$ & $17,1(11,6)$ & 0,002 \\
\hline Insuficiencia de fondos o tiempo protegido para la investigación & $19,6(12,2)$ & $17,9(11,3)$ & 0,351 \\
\hline $\begin{array}{l}\text { Insuficiencia de tiempo protegido para la educación o } \\
\text { la capacitación médica continuas }\end{array}$ & $20,8(10,8)$ & $16,3(12,1)$ & 0,007 \\
\hline Participación en el programa de mentor-discípulo & $20,8(11,6)$ & $18,0(11,6)$ & 0,017 \\
\hline Falta de agradecimiento de los pacientes o sus padres & $22,4(14,0)$ & $17,3(10,5)$ & 0,021 \\
\hline Falta de reconocimiento de sus supervisores & $22,3(11,3)$ & $15,9(11,2)$ & $<0,001$ \\
\hline $\begin{array}{l}\text { Falta de instalaciones, como una estación de trabajo o } \\
\text { un espacio para los médicos de guardia }\end{array}$ & $20,6(12,0)$ & $17,0(11,2)$ & 0,031 \\
\hline Falta de incentivos y promociones & $21,9(12,0)$ & $15,8(10,6)$ & $<0,001$ \\
\hline Falta de oportunidades de capacitación profesional & $20,0(11,3)$ & $17,1(11,8)$ & 0,074 \\
\hline Falta de recursos cuando los equipos están defectuosos & $20,1(11,6)$ & $15,3(11,0)$ & 0,006 \\
\hline Barrera idiomática con los pacientes & $20,7(12,0)$ & $17,4(11,3)$ & 0,072 \\
\hline Conflictos entre enfermeras y médicos & $21,4(11,4)$ & $16,4(11,4)$ & 0,003 \\
\hline Problemas de salud física o mental & $23,9(12,7)$ & $17,1(10,8)$ & 0,003 \\
\hline $\begin{array}{l}\text { Establecimiento de metas inaccesibles o } \\
\text { autoimposición de ese tipo de metas }\end{array}$ & $21,7(12,1)$ & $17,5(11,3)$ & 0,004 \\
\hline Trabajo bajo presión del tiempo y necesidad de cumplir con los plazos & $22,9(11,3)$ & $14,2(10,3)$ & $<0,001$ \\
\hline Trámites burocráticos y administrativos innecesarios & $20,3(12,0)$ & $15,8(10,5)$ & 0,006 \\
\hline $\begin{array}{l}\text { Vida personal y familiar afectada por las exigencias laborales/ } \\
\text { falta de equilibrio entre la carrera y la familia }\end{array}$ & $21,3(11,6)$ & $14,2(10,4)$ & $<0,001$ \\
\hline Exceso de trabajo durante las guardias y horarios prolongados de trabajo & $20,5(11,2)$ & $15,0(11,6)$ & 0,002 \\
\hline Subordinados/colegas incompetentes & $19,6(11,6)$ & $17,6(11,6)$ & 0,229 \\
\hline
\end{tabular}


grado de estrés percibido. ${ }^{23}$ La versión malasia de la EEP-10 fue una medida validada del estrés en la población malasia con propiedades psicométricas satisfactorias y un coeficiente alfa de Cronbach de 0,71. ${ }^{22,23}$ El coeficiente alfa de Cronbach de la escala de estrés percibido utilizada en este estudio era de 0,843 .

\section{Consideraciones éticas}

Este protocolo de investigación y su financiación fueron registrados y aprobados por la Comisión Conjunta en Investigación y Ética de la Universidad Médica Internacional (CSc/Sem6(36)2014) y el Comité de Ética en la Investigación Clínica del Ministerio de Salud de Malasia (NMRR-15-144-24112). Los fondos para este estudio, incluidos los incentivos en efectivo que se entregaron como muestra de agradecimiento, provienen de una beca para investigación de la Universidad Médica Internacional (CSc/Sem6(36)2014). Se dio a los participantes una breve explicación de los objetivos del estudio, se les garantizó la confidencialidad y se obtuvo su consentimiento.

\section{Análisis estadístico}

El análisis de los datos de este estudio se realizó con el paquete estadístico para ciencias sociales (SPSS), versión 16.0. Se hizo un análisis descriptivo de todas las variables del estudio. Para obtener el puntaje total correspondiente al AE se sumaron los 9 puntos de la subescala de AE. Para obtener el puntaje total correspondiente a la EEP se sumaron los 10 puntos de la EEP. Se evaluó la normalidad para cada variable continua. El puntaje total para el AE se categorizó en bajo, moderado y alto para obtener las frecuencias. Para validar la subescala de AE se llevaron a cabo un análisis de confiabilidad y un análisis factorial exploratorio. Para comparar la media del puntaje de agotamiento emocional correspondiente a todas las variables sociodemográficas y fuentes de estrés, se emplearon una prueba $t$ y un análisis de varianza (ANOVA). La correlación entre la EEP y el AE se evaluó con el coeficiente de correlación de Pearson. Para obtener los predictores más importantes del agotamiento emocional se usó la regresión lineal múltiple. Se verificaron la colinealidad y otras suposiciones, y se estableció un valor de $p<0,05$ como el nivel de significación.

\section{RESULTADOS \\ Características sociodemográficas}

Ciento noventa y siete participantes devolvieron los cuestionarios completos (tasa de respuesta del 65\%). Los participantes que respondieron fueron predominantemente mujeres $(72,6 \%)$, casados $(52,3 \%)$, malasios $(47,2 \%)$, menores de 30 años $(61,4 \%)$ y graduados en el extranjero $(59,4 \%)$. La mayoría eran médicos $(47,2 \%)$ y contaban con una experiencia laboral inferior a dos años $(61,9 \%)$ (Tabla 1).

\section{Fuentes de estrés laboral mencionadas por los participantes que respondieron}

Las fuentes más habituales de estrés mencionadas fueron trato con padres difíciles $\square$ $(80,2 \%)$; sensación de que el nivel de conocimiento no es suficiente para satisfacer las demandas laborales $\square(72,6 \%)$; "salas repletas de pacientes" $(70,6 \%)$; "temor a tener problemas legales relacionados con el ejercicio de la medicina" $(70,1 \%)$ y atención de niños con cuadros agudos en cuidados intensivos $\square(68,5 \%)$.

TABLA 3: Análisis multifactorial: predictores del agotamiento emocional

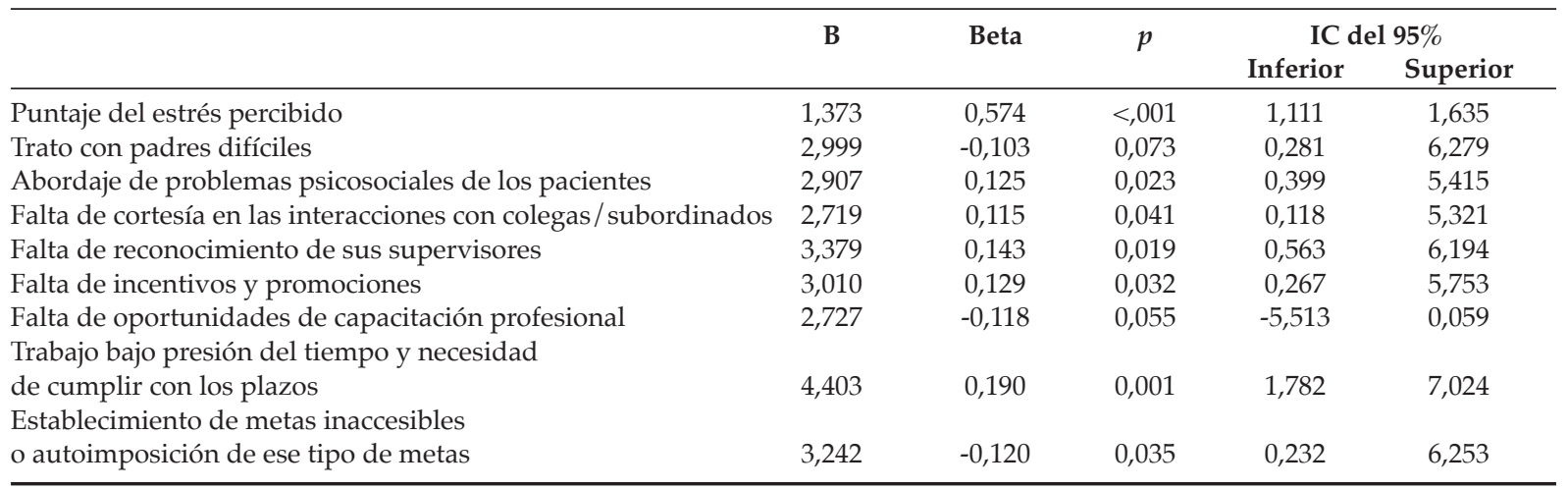

B: cálculo de la regresión; Beta: coeficiente estandarizado; IC: intervalo de confianza 


\section{Validez factorial y consistencia interna del AE}

La validez factorial del AE se examinó mediante un análisis factorial exploratorio, que mostró que la versión en inglés del AE utilizada en este estudio es unifactorial, lo que refuerza la idea de que el $\mathrm{AE}$ es un componente. El coeficiente alfa de Cronbach para los 9 puntos del AE fue de 0,93, lo cual indica que la subescala de $\mathrm{AE}$ es un instrumento válido y confiable para medir el burnout emocional $\square$ entre los médicos malasios.

\section{El agotamiento emocional y los factores asociados}

Mediante el uso de la subescala de AE del MBI-HSS, se sumaron los puntajes brutos y luego se categorizó el AE como bajo $(\leq 16)$, moderado (de 17 a 26$)$ y alto $(\geq 27){ }^{21,24}$ El AE medio entre los médicos fue de $18,5 \pm 11,6$, y el puntaje para el $\mathrm{AE}$ osciló entre 0 y 48.

Cincuenta médicos $(25,4 \%)$ mostraron un nivel de agotamiento emocional alto, $48(24,4 \%)$, mostraron un nivel moderado y $99(50,2 \%)$, un nivel bajo. Para examinar los factores asociados con el agotamiento emocional en un análisis bivariante, usamos el puntaje total del agotamiento emocional como variable continua. No hubo una asociación importante entre el agotamiento emocional y las variables sociodemográficas $(p>0,05)$ (Tabla 1). Para examinar la asociación entre el agotamiento emocional y las fuentes percibidas de estrés laboral, se comparó la media del puntaje total de agotamiento emocional entre las personas que respondieron Sí y las que respondieron No a cada fuente de estrés. En la Tabla 2 puede observarse que 29 de los 38 puntos mostraron una asociación importante con el agotamiento emocional $(p<0,05)$, con un puntaje medio para el AE más alto entre los que respondieron Sí que entre los que respondieron No. El análisis de correlación de Pearson mostró una correlación positiva y significativa entre la EEP y el agotamiento emocional $(\mathrm{r}=0,625 ; p<0,001)$.

En el análisis multifactorial (Tabla 3), se incluyeron todas las variables que mostraron una asociación importante con el agotamiento emocional. El $\mathrm{R}^{2}$ ajustado de 0,48 indicó que el modelo explica el $48 \%$ de la variabilidad del agotamiento emocional. El modelo total fue significativo $(p<0,05)$. Los estadísticos de colinealidad no muestran colinealidad entre las variables porque los valores de tolerancia y factor de inflación de la varianza se encuentran dentro de los valores recomendados.

\section{DISCUSIÓN}

El inventario MBI-HSS estuvo compuesto por tres subescalas: $\mathrm{AE}$, despersonalización y logro personal. ${ }^{1}$ Nosotros empleamos la subescala de AE para medir el "burnout emocional" porque es la dimensión de burnout más significativa en el MBI. La dimensión AE se refiere a la sensación de estar extenuado y cansado emocional y psicológicamente a causa del trabajo. ${ }^{25}$ Según consta en la bibliografía, el AE es un elemento fundamental del burnout y la dimensión más frecuentemente informada y analizada de las tres subescalas del burnout. Maslach et al. informan que cuando las personas dicen de sí mismas que están sufriendo burnout, la mayoría de las veces se refieren al agotamiento emocional. ${ }^{26}$ Además, se ha demostrado que el $\mathrm{AE}$ genera despersonalización y cinismo. ${ }^{27}$

Este es el primer estudio en Malasia en investigar los factores asociados con el agotamiento emocional en el contexto de la pediatría. Dada la frecuencia, la duración y la intensidad emocional de las interacciones clínicas bidireccionales entre los niños y sus cuidadores, los pediatras están en mayor riesgo de burnout profesional. $^{28}$

De los 197 médicos encuestados, el 25,4\% mostró un alto nivel de agotamiento emocional. Se informó una tasa similar entre los pediatras de Argentina. ${ }^{29}$ Las tasas de prevalencia fueron inferiores en Arabia Saudita (34\%), ${ }^{4}$ pero más altas en Alemania (10\%). ${ }^{28}$

En este estudio, encontramos que existían asociaciones importantes entre el agotamiento emocional y el estrés percibido, la gestión clínica y los factores relacionados con el entorno laboral. Las cifras informadas de pediatras afectados varían de un país a otro. La mayoría de los estudios se basan sobre la hipótesis de que el burnout emocional de los pediatras está asociado con las características demográficas, el entorno laboral y los recursos en los hospitales, la gestión clínica o factores personales. ${ }^{28}$ Los datos demográficos informados en este estudio no mostraron asociaciones importantes con el agotamiento emocional. Los hallazgos informados recientemente son similares. ${ }^{30}$ La pediatría, una práctica multidisciplinaria que incluye atención de emergencia e intensiva, pediatría general, neonatología y subespecialidades, genera una carga de trabajo demandante a nivel físico y mental. ${ }^{31}$ Hubo una asociación importante entre los problemas psicosociales del paciente y el agotamiento emocional, que 
podría deberse al hecho de que los profesionales deben explicar tediosamente una y otra vez las necesidades de tratamiento de los pacientes, los posibles efectos secundarios, la interrupción de la asistencia a clases y de sus rutinas sociales, y la suspensión de las actividades recreativas, y a que deben aliviar la ansiedad de los cuidadores por asimilar la información sobre el tratamiento. ${ }^{32}$ Un considerable nivel de estrés laboral genera mayor agotamiento y quita energías para el trabajo diario. ${ }^{31}$ Este estudio mostró una correlación significativa entre el estrés laboral y el agotamiento emocional de los pediatras. Weigl et al., informaron datos parecidos. ${ }^{28}$

Es necesario mencionar algunas limitaciones de este estudio. En primer lugar, su diseño transversal obstaculiza la posibilidad de establecer una relación causa-efecto, a diferencia de lo que pudieron lograr Pantaleoni et al. ${ }^{5}$ Por otro lado, los médicos que intervinieron en este estudio eran residentes y pediatras, y por lo tanto su experiencia y su tiempo de exposición a la disciplina de la pediatría eran diferentes. La enorme cantidad de médicos graduados en los últimos años se ve reflejada en el alto porcentaje de médicos pediatras jóvenes sin experiencia. ${ }^{33} \mathrm{~A}$ fin de abordar esta limitación, usamos análisis univariantes y multivariantes para controlar los factores de confusión. Otro tema para tener en cuenta es que, mientras se realizaba el estudio, los médicos fueron asignados a diferentes rotaciones (por ejemplo, de cuidados intensivos a atención de pacientes ambulatorios), de modo que experimentaron distintas cargas de trabajo. Esto pudo influir en su actitud frente al trabajo y en sus respuestas al cuestionario.

Pese a estas limitaciones, este estudio ofrece una visión más profunda de la educación continua y la capacitación de los médicos pediatras. Los resultados registrados muestran asociaciones importantes entre una gran cantidad de factores de estrés percibidos relacionados con el trabajo y el agotamiento emocional de los pediatras. Las presiones en el rendimiento, que imponen limitaciones de tiempo, estuvieron estrechamente asociadas con el agotamiento emocional de los pediatras. De manera similar, los factores relacionados con el entorno laboral, como el establecimiento de metas inaccesibles y la falta de cortesía en las interacciones con colegas o subordinados, estuvieron estrechamente asociados con el agotamiento emocional. Estos resultados son similares a los obtenidos en estudios anteriores. ${ }^{21,24,34}$ Aunque existen pocas pruebas prospectivas del efecto que los factores estresantes del contexto institucional tienen sobre el agotamiento emocional de los pediatras, los estudios realizados en otras especialidades médicas corroboran que el desequilibrio entre el esfuerzo y la recompensa tienen un impacto en el burnout. ${ }^{28}$ Independientemente de los últimos ajustes en los salarios, ${ }^{24}$ la desproporción en las remuneraciones y la falta de incentivos, promociones y reconocimiento de parte de los supervisores continúan siendo factores importantes asociados con el agotamiento emocional, tanto en este estudio como en estudios similares anteriores. ${ }^{24,28,35} \mathrm{El}$ modelo desequilibrio esfuerzorecompensa $\square$ exige que el equilibrio entre los esfuerzos hechos en el trabajo y las recompensas recibidas se ejecute a través de tres dominios: salario, prestigio y seguridad en el empleo. ${ }^{24,28}$

En conclusión, estos datos indican que el agotamiento emocional de los médicos de los departamentos de pediatría de los hospitales públicos es alto y que se relaciona con fuentes de estrés provenientes del entorno laboral y con el nivel de estrés autopercibido, pero no con factores sociodemográficos. Por lo tanto, debería abordarse el análisis y el correcto control de estas fuentes de estrés de manera que pueda mejorarse el entorno laboral de los pediatras y, por consiguiente, su eficiencia y satisfacción con el trabajo.

\section{REFERENCIAS}

1. Maslach C, Jackson SE. The measurement of experienced burnout. J Occup Health 1981;2:99-113.

2. Pistelli Y, Perochena J, Moscoloni N, Tarrés MC.Síndromede desgaste profesionalen médicos pediatras. Análisis bivariado y multivariado. Arch Argent Pediatr 2011;109(2):129-34.

3. Martins AE, Davenport MC, Del Valle MP, et al. Impact of a brief intervention on the burnout levels of pediatric residents. J Pediatr (Rio J) 2011;87(6):493-8.

4. Al-Youbi RA, Jan MM. Burnout syndrome in pediatric practice. Oman Med J 2013;28(4):252-4.

5. Pantaleoni JL, Augustine EM, Sourkes BM, Bachrach LK. Burnout in pediatric residents over a 2-year period: a longitudinal study. Acad Pediatr 2014;14(2):167-72.

6. Wright JG, Khetani N, Stephens D. Burnout among faculty physicians in an academic health science centre. Paediatr Child Health 2011;16(7):409-13.

7. Cavaliere TA, Daly B, Dowling D, Montgomery K. Moral distress in neonatal intensive care unit RNs. Adv Neonatal Care 2010;10(3):145-56.

8. ConradD, Kellar-Guenther Y.Compassion fatigue, burnout, and compassion satisfaction among Colorado child protection workers. Child Abuse Negl 2006;30(10):1071-80.

9. Roth M, Morrone K, Moody K, Kim M, et al. Career burnout among pediatric oncologists. Pediatr Blood Cancer 2011;57(7):1168-73.

10. Jan MM. Perception of pediatric neurology among nonneurologists. J Child Neurol 2004;19(1):1-5. 
11. Branco RG, Garcia TT, Molon ME, Garcia PCR, et al. 1138 Burnout Syndrome Among Pediatricians: A Case Control Study Comparing Pediatric Intensivists and General Pediatricians. Pediatr Res 2010;68:564.

12. Passalacqua SA, Segrin C. The effect of resident physician stress, burnout, and empathy on patient-centered communication during the long-call shift. Health Commun 2012;27(5):449-56.

13. Shanafelt TD, Balch CM, Bechamps G, Russell T, et al. Burnout and medical errors among American surgeons. Ann Surg 2010;251(6):995-1000.

14. Piko BF. Burnout, role conflict, job satisfaction and psychosocial health among Hungarian health care staff: A questionnaire survey. Int J Nurs Stud 2006;43(3):311-8.

15. BrownSD, Goske MJ, Johnson CM. Beyond substance abuse: stress, burnout, and depression as causes of physician impairment and disruptive behavior. J Am Coll Radiol 2009;6(7):479-85.

16. Dyrbye LN, Thomas MR, Massie FS, Power DV, et al. Burnout and suicidal ideation among U.S. medical students. Ann Intern Med 2008;149(5):334-41.

17. Sonneck G, Wagner R. Suicide and burnout of physicians. OMEGA-J Death Dying 1996;33:255-63.

18. Rogers AC. Vulnerability, health and health care. J Adv Nurs 1997;26(1):65-72.

19. The World Bank: World Development Indicators 2015. [Acceso: 30 de enero de 2016] Disponible en: http:/ / data. worldbank.org/indicator

20. Registro Nacional de Especialistas: Malasia. General Paediatrics 2015. [Acceso: 20 de enero de 2016] Disponible en: https: / / www.nsr.org.my / list1view.asp?page=1

21. Al-Dubai SAR, Rampal KG. Prevalence and associated factors of burnout among doctors in Yemen. Field Study. J Occup Health 2010;52(1):58-65.

22. Al-Dubai SAR, Al-Shaggaa MA, Rampal KG, Sulaiman NA. Factor structure and reliability of the Malay version of Perceived Stress Scale among medical students. Malays J Med Sci 2012;19(3):43-9.

23. Al-Dubai SAR, Ganasegeran K, Barua, Rizal AAM, et al, Evaluation of psychometric properties of the Malay version Perceived Stress Scale in two occupational settings in Malaysia. Ann Med Health Sci Res 2014;4(Suppl 2):S104-7.
24. Al-Dubai SAR, Ganasegeran K, Perianayagam W, Rampal KG. Emotional Burnout, Perceived Sources of Job Stress, Professional Fulfillment, and Engagement among Medical Residents in Malaysia. ScientificWorldJournal 2013;2013:137620.

25. Lambert EG, Barton-Bellessa SM, Hogan NL. The Consequences of Emotional Burnout Among Correctional Staff. Sage Open 2015;5(2):1-15.

26. Maslach C, Schaufeli W, Leiter M. Job burnout. Annu Rev Psychol 2001;52:397-422.

27. Maslach C, Leiter M. Early predictors of job burnout and engagement. J Appl Psychol 2008;93(3):498-512.

28. Weigl M, Schneider A, Hoffmann F, Angerer P. Work stress, burnout, and perceived quality of care: a crosssectional study among hospital paediatricians. Eur J Pediatr 2015;174(9):1237-46.

29. Galván ME, Vassallo JC, Rodríguez SP, Otero P, et al. Síndrome de desgaste profesional (burnout) en médicos de unidades de cuidados intensivos pediátricos en la Argentina. Arch Argent Pediatr 2012;110(6):466-73.

30. Aksoy DY, Durusu Tanriover M, Unal S, Dizdar O, et al. Burnout syndrome during residency in internal medicine and pediatrics in a country without working time directive. Int J Health Care Qual Assur 2014;27(3):223-30.

31. Pantaleoni JL, Augustine EM, Sourkes BM, Bachrach LK. Burnout in Pediatric Residents Over a 2-Year Period: A Longitudinal Study. Acad Pediatr 2014;14(2):167-72.

32. Kohlsdorf M, Costa Jr AL. Psychosocial Impact of Pediatric Cancer on Parents: A Literature Review. Paidéia (Riberãon Preto) 2012;22(51):119-29.

33. Quek DKL. "The Standards of Medical Education in Malaysia and Its Acceptability. Medico-Legal Society of Malaysia Conference. Royale Chulan Hotel, Kuala Lumpur. 16 Nov. 2011. [Acceso: 25 de octubre de 2016] Disponible en: http:/ / www.mpsr.org/ 2011/12/the-standards-ofmedical-education-in-malaysia-and-its-acceptability /

34. Yusoff MSB, Jie TY, Esa AR. Stress, stressors and coping strategies among house officers in a Malaysian hospital. ASEAN Journal of Psychiatry 2011;12(1):1-10.

35. Bowden MJ, Mukherjee S, Williams LK, DeGraves S, et al. Work-related stress and reward: an Australian study of multidisciplinary pediatric oncology healthcare providers. Psychooncology 2015; 24(11):432-8. 